\title{
Reversible ventriculoperitoneal shunt dysfunction and chronic constipation: case report
}

\author{
Barbara A. Morais, MD, Daniel D. Cardeal, MD, Fernanda G. Andrade, PhD, \\ Wellingson S. Paiva, PhD, Hamilton Matushita, PhD, and Manoel J. Teixeira, PhD \\ Department of Neurosurgery, School of Medicine, University of São Paulo, São Paulo, Brazil
}

\begin{abstract}
Constipation can cause transient malfunction of the ventriculoperitoneal shunt (VPS). Patients with myelomeningocele or cerebral palsy are often diagnosed with hydrocephalus and constipation due to neurogenic bowel. These patients are more prone to VPS dysfunction, often requiring surgical revision. The authors report the case of a 6 -year-old girl with a VPS that had been implanted due to hydrocephalus secondary to myelomeningocele. The patient was brought to the emergency department with intermittent headache, vomiting, constipation, and abdominal distension and pain. A CT scan revealed ventricular dilatation and radiography of the abdomen showed bowel loop distension. After a Fleet enema and digital maneuvers, her abdominal distension and symptoms improved. A CT scan obtained 24 hours later showed a reduction in ventricular size. The mechanism by which constipation can lead to VPS malfunction can be traced to indirect increases of intraabdominal pressure and direct obstruction of the catheter by distended intestinal loops. Treating constipation can restore the free circulation of the CSF and avoid surgical intervention. Careful neurological monitoring of these patients is essential, because some measures used to treat constipation can increase intracranial pressure. The objective of this report was to highlight constipation as a possible cause of transient VPS malfunction, thereby avoiding unnecessary surgical revisions, to which children with hydrocephalus are frequently submitted.
\end{abstract}

https://thejns.org/doi/abs/10.3171/2018.2.PEDS17574

KEYWORDS constipation; hydrocephalus; malfunction; ventriculoperitoneal shunt; myelomeningocele

$\mathrm{H}$ YDROCEPHALUS affects both pediatric and adult populations, with an estimated prevalence in the general population of between $1.0 \%$ and $1.5 \% .^{11}$ Ventriculoperitoneal shunts (VPSs) continue to be the standard treatment for hydrocephalus, despite the high rates of failure associated with these devices and advances in the field. One-year failure rates for VPSs for hydrocephalus are reported to be approximately $40 \%$ - this failure rate increases to $70 \%$ after 10 years of follow-up. In 2013, Stone et al. reported that $84 \%$ of pediatric patients with a VPS followed for 15 years required a revision. ${ }^{12}$ Importantly, in their study, Stone et al. found that catheter occlusion was the primary reason for VPS revision, with no specific etiology of hydrocephalus, valve type, or patient demographic characteristic predicting the need for VPS revision during the 15 years following its placement.

The VPS is based on the pressure differential between the ventricular space and the peritoneal cavity. Although VPS dysfunction can occur for a variety of reasons, approximately $25 \%$ of cases are related to complications at

ABBREVIATIONS VPS $=$ ventriculoperitoneal shunt. 

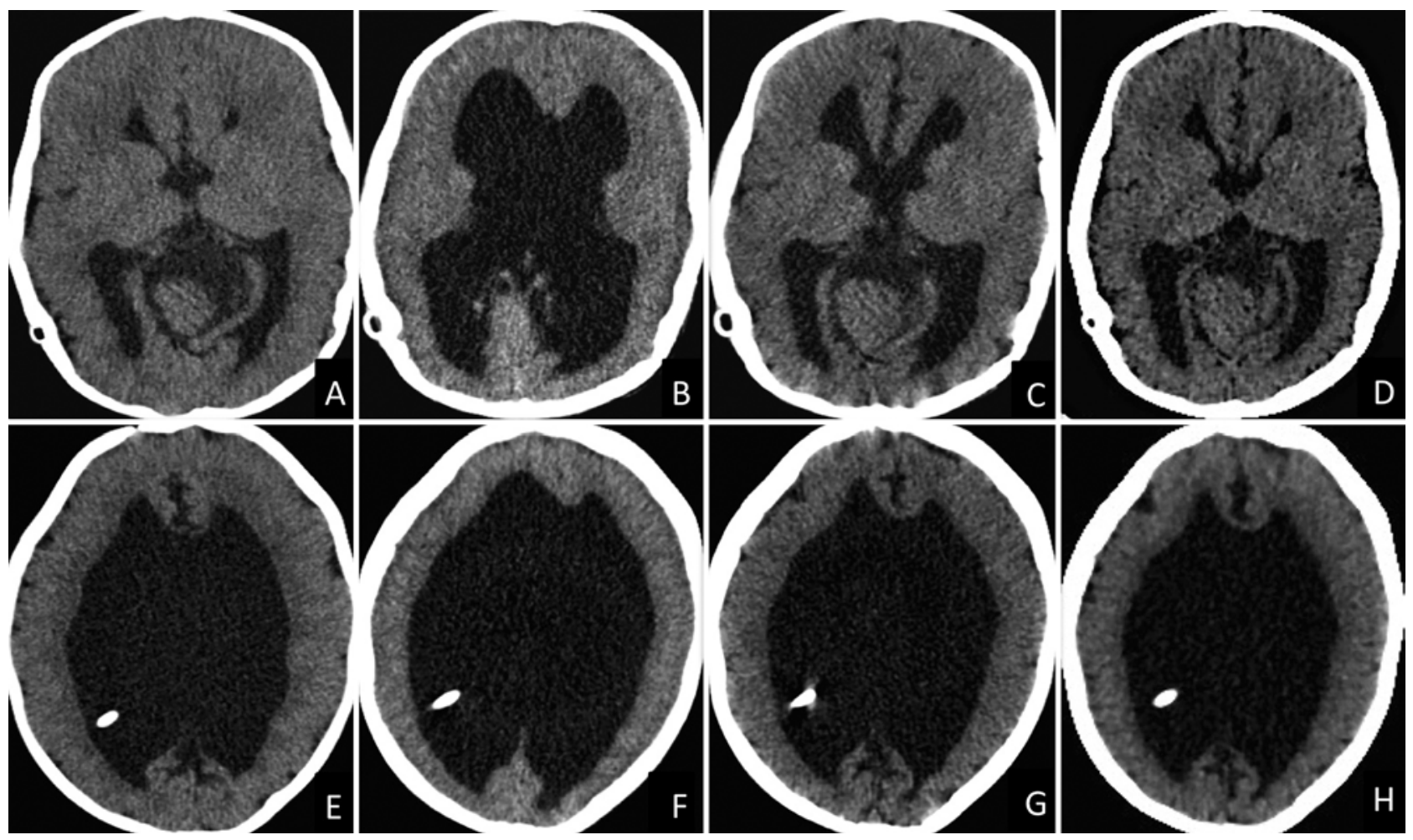

FIG. 1. Brain CT scans, axial images. A and E: Baseline ventricles. B and F: Scans obtained during a constipation episode, with moderate ventricular enlargement. C and G: Scans obtained 24 hours after clinical treatment, with progressive ventricular size reduction. D and $\mathrm{H}$ : Scans obtained 1 month after clinical treatment, with return to baseline.

the abdominal end of the shunt. ${ }^{1}$ In particular, increases in intraabdominal pressure can induce shunt failure by decreasing the drainage of CSF from the ventricles. ${ }^{4}$ Constipation, usually regarded as a trivial condition, can play an important role in shunt failure, either by mechanically blocking draining from the peritoneal end of the VPS catheter, or by increasing intraabdominal pressure.

Chronic constipation secondary to neurogenic bowel is common in patients with spinal dysraphism and cerebral palsy. ${ }^{9}$ Here we report on a 6-year-old patient with myelomeningocele who suffered transitory VPS failure, which resolved after her constipation was relieved, avoiding unnecessary surgery.

\section{Case Report}

History

A 6-year-old girl was brought to the emergency department with intermittent headache, lethargy, nausea, vomiting, and abdominal distension and pain. The patient had no seizures, fever, or loss of consciousness. The girl's history revealed a previous diagnosis of hydrocephalus, secondary to myelomeningocele, which had been treated with placement of a VPS at birth. She also had a history of neurogenic bladder and bowel with chronic constipation, both of which had been treated clinically.

\section{Examination}

Brain CT scans (Fig. 1B and F) revealed a moderate ventricular dilatation, compared to her previous scans (Fig.
$1 \mathrm{~A}$ and E). Shunt survey showed no evidence of disconnection in the shunt assembly. Radiography of the abdomen (Fig. 2) showed dilated bowel loops and a large stool burden.

\section{Treatment and Posttreatment Course}

Attempts to relieve the stool burden with a pediatric Fleet enema were ineffective, but release of bowel contents was achieved after digital removal of the feces. Following removal of the feces, abdominal distension and other symptoms improved. Brain CT scanning performed 24 hours later showed a reduction in ventricular size (Fig. 1C and G). Since the incident, she has been managed on an appropriate bowel regimen to prevent constipation. One month later, follow-up brain CT revealed that ventricles had returned to baseline (Fig. 1D and H). Fourteen months later, at outpatient follow-up, the patient was asymptomatic and doing well.

\section{Discussion}

The association between hydrocephalus and myelomeningocele has been well documented and is present in almost $80 \%$ of patients. More than $90 \%$ of these children are also affected by neurogenic bowel and tend to be chronically constipated. ${ }^{1}$ Neurogenic bowel is characterized by inefficient peristalsis, a loss of afferent sensory input signaling fullness of the rectum, loss of motor innervation to the anal sphincter, and an inability to contract the muscles of the pelvic floor. ${ }^{3}$ Also, the use of anticholinergic medi- 
cations (which are often used to treat neurogenic bladder in these patients), is a predisposing factor for aggravation of their bowel dysfunction.

The first study proposing constipation as a possible factor for VPS malfunction was published by Bragg et al. in 1994. ${ }^{1}$ In their case series, ${ }^{2}$ the authors reported on 51 children with VPS malfunction, and the need for shunt revision was proportionally similar for children with and without constipation. Although discussed in their article, the authors could not prove that VPS malfunction and constipation were related. However, since then there have been a number of reports describing transitory dysfunction of VPSs in both pediatric and adult patients with chronic constipation. 4,6,7,9,11 Transient VPS dysfunction has already been described in children with other abdominal issues such as urinary bladder infection. ${ }^{13}$ As Tubbs et al. discuss, abdominal CSF can behave as an ascitic fluid, even in the absence of peritoneal accumulation; therefore, it can predispose patients to spontaneous bacterial peritonitis. In addition, patients with abdominal conditions involving inflammation or infection (such as a urinary tract infection or even significant constipation) may have a predisposition to translocation of bacteria. ${ }^{13}$

It is well known ${ }^{12}$ that managing hydrocephalus becomes increasingly complex after repeated shunt failures. Children who undergo multiple shunt revisions can be predisposed to gliosis and multiloculated ventricles, peritoneal fibrotic adhesions, pseudocyst formation, and CSF ascites. Therefore, this case highlights the importance of recognizing the role of chronic constipation as a cause of VPS failure that is reversible when appropriate clinical treatment is performed. However, conservative treatment requires close neurological monitoring because shunt-dependent patients with hydrocephalus can deteriorate rapidly. In addition, the use of a bowel enema in the presence of papilledema can sometimes precipitate an acute rise in intracranial pressure.

Many variables associated with shunt failure have already been identified. Because of this, we would like to reinforce that the mere presence of constipation should not delay the search for other more common causes of shunt obstruction. Although the actual incidence of VPS failure secondary to constipation is unknown, studies suggest that the reductions in patient morbidity and health care costs may be considerable. ${ }^{11}$

Currently, little is known about the correlation between constipation as an isolated cause of intracranial hypertension in the pediatric population. Although other pathologies such as idiopathic intracranial hypertension are likely to be involved, researchers have not been able to correlate them to gut motility dysfunction. In 2016, Prasad et al. reported the case of a severely constipated child with secondary posterior reversible encephalopathy syndrome. However, in this case, the encephalopathy was probably due to a secondary renovascular hypertension and not only to the constipation itself. ${ }^{10}$

On the other hand, it has been reported that elevated intracranial pressure can influence gut motility. ${ }^{3}$ Increases in intracranial pressure induce massive increases in sympathetic activity, which can be responsible for many of the gastrointestinal symptoms that patients display, such as

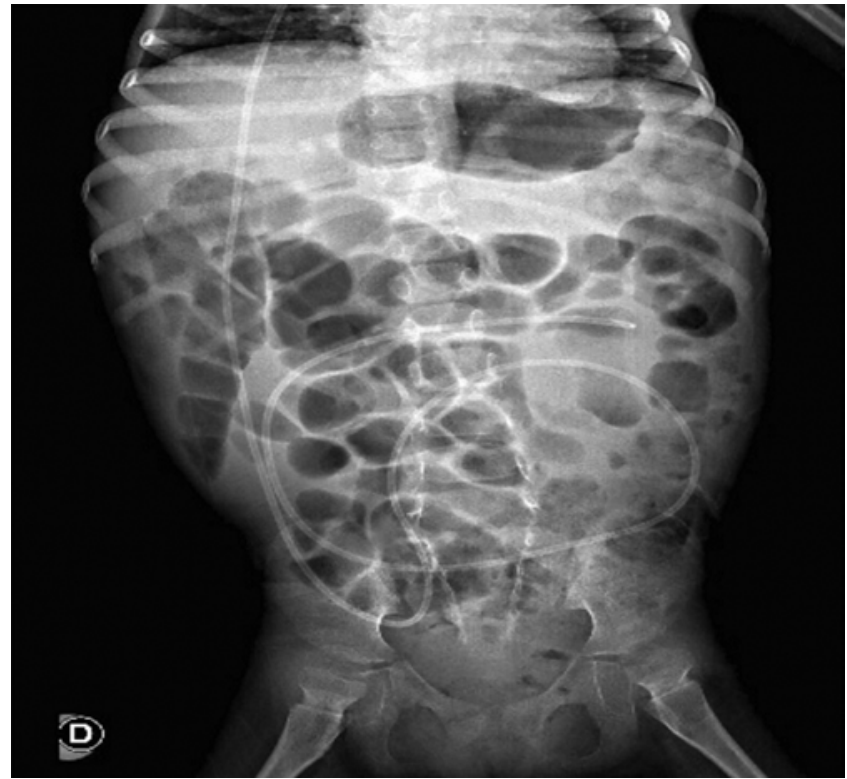

FIG. 2. Anteroposterior radiography of the patient's abdomen showing dilated colonic loops filled with fecal contents suggestive of significant constipation.

hypersecretion of gastric acid and gastric hypomotility., 3,5 To help distinguish whether the patient's constipation was a consequence of or the trigger for elevated intracranial pressure, it is important that the evaluation include an investigation of his or her background and the sequence of clinical events.

\section{Conclusions}

Our aim in presenting this case was to draw the attention of the attending physician to this condition as a reversible cause of VPS malfunction. For patients with neurological diseases (e.g., those with dysraphism and cerebral palsy) in whom associated conditions like hydrocephalus and chronic constipation are a daily reality, our purpose is to avoid unnecessary surgical valve revisions, to which children with hydrocephalus are often submitted.

\section{References}

1. Bragg CL, Edwards-Beckett J, Eckle N, Principe K, Terry D: Shunt dysfunction and constipation: could there be a link? J Neurosci Nurs 26:91-94, 1994

2. Bragg CL, Edwards-Beckett J, Eckle N, Principe K, Terry D: Ventriculoperitoneal shunt dysfunction and constipation: a chart review. J Neurosci Nurs 26:265-269, 1994

3. Lucena AF, Tibúrcio RV, Vasconcelos GC, Ximenes JDA, Cristino Filho G, Graça RV: Influence of acute brain injuries on gut motility. Rev Bras Ter Intensiva 23:96-103, 2011

4. Martínez-Lage JF, Martos-Tello JM, Ros-de-San Pedro J, Almagro MJ: Severe constipation: an under-appreciated cause of VP shunt malfunction: a case-based update. Childs Nerv Syst 24:431-435, 2008

5. Matthews DE, Heimansohn DA, Papaila JG, Lopez R, Vane DW, Grosfeld JL: The effect of increased intracranial pressure (ICP) on gastric motility. J Surg Res 45:60-65, 1988

6. Miele VJ, Bendok B, Bloomfield SM, Ondra SL, Bailes JE: Ventriculoperitoneal shunt dysfunction in adults secondary 
to conditions causing a transient increase in intra-abdominal pressure: report of three cases. Neurosurgery 55:434, 2004

7. Mirzayan MJ, Koenig K, Bastuerk M, Krauss JK: Coma due to meteorism and increased intra-abdominal pressure subsequent to ventriculoperitoneal shunt dysfunction. Lancet 368:2032, 2006

8. Mulvihill SJ, Pappas TN, Debas HT: Effect of increased intracranial pressure on gastric acid secretion. Am J Surg 151:110-116, 1986

9. Muzumdar D, Ventureyra ECG: Transient ventriculoperitoneal shunt malfunction after chronic constipation: case report and review of literature. Childs Nerv Syst 23:455-458, 2007

10. Prasad M, Wetzler G, Holtmann J, Dapul H, Kupferman JC: Constipation, renovascular hypertension, and posterior reversible encephalopathy syndrome (PRES). Eur J Pediatr 175:421-425, 2016

11. Powers CJ, George T, Fuchs HE: Constipation as a reversible cause of ventriculoperitoneal shunt failure. Report of two cases. J Neurosurg 105 (3 Suppl):227-230, 2006

12. Stone JJ, Walker CT, Jacobson M, Phillips V, Silberstein HJ: Revision rate of pediatric ventriculoperitoneal shunts after 15 years. J Neurosurg Pediatr 11:15-19, 2013
13. Tubbs RS, Wellons JC III, Blount JP, Oakes WJ: Transient ventriculoperitoneal shunt dysfunction in children with myelodysplasia and urinary bladder infection. Report of three cases. J Neurosurg 102 (2 Suppl):221-223, 2005

\section{Disclosures}

The authors report no conflict of interest concerning the materials or methods used in this study or the findings specified in this paper.

\section{Author Contributions}

Conception and design: Morais, Matushita. Acquisition of data: Morais, Cardeal, Andrade, Matushita. Drafting the article: Morais. Critically revising the article: Paiva, Matushita, Teixeira. Reviewed submitted version of manuscript: Morais, Paiva, Matushita, Teixeira.

\section{Correspondence}

Barbara A. Morais: School of Medicine, University of São Paulo, Brazil.babyamorais@gmail.com. 